\title{
Phylogenetic relationships and genetic diversity of the Polypedates leucomystax complex in Thailand
}

\author{
Kittisak Buddhachat ${ }^{\text {Corresp., }}{ }^{1}$, Chatmongkon Suwannapoom ${ }^{2}$ \\ 1 Department of Biology, Naresuan University, Phitsanulok, Thailand \\ ${ }^{2}$ School of Agriculture and Natural Resources, University of Phayao, Phayao, Thailand \\ Corresponding Author: Kittisak Buddhachat \\ Email address: kittisakbu@nu.ac.th
}

Taxonomic uncertainty of the Asian tree frog Polypedates leucomystax complex presents the challenging task of inferring its biogeographical history. Here, we describe its dispersion and the genetic relationships among different populations in Thailand, where we connect the population of the P. leucomystax complex of the Sunda Islands to the Indochina (mainland) population based on analyses of 266 sequences of the mitochondrial cytochrome c oxidase subunit I (COI) gene. Our maternal genealogy implies that there are four well-supported lineages in Thailand, consisting of Northern A (clade A: Polypedates sp.), Nan (clade B: P. cf. impresus), Southern (clade C: P. cf. leucomystax) and Northern D (clade D: $P$. cf. megacephalus), with Bayesian posterior probability $>0.9$. Phylogeny and haplotype networks indicate that clades A, B and D are sympatric. In contrast, clade $C(P$. cf. leucomystax) and clade D (P. cf. megacephalus) are genetically divergent due to the geographical barrier of the Isthmus of Kra, resulting in an allopatric distribution. Climatic conditions, in particular differences in rainfall on each side of the Isthmus of Kra, may play an important role in limiting the immigration of both clades. For the within-populations of either clades $C$ or $D$, there was no significant correlation between geographic and genetic distance by the isolation-by-distance test, indicating intraspecific-dispersal of each clade. Population expansion occurred in clade C, whereas clade D showed a constant population. Taken together, the $P$. leucomystax complex in South East Asia may have diversified under climatic pressure, leading to allopatric and/or sympatric speciation. 
1 Phylogenetic relationships and genetic diversity of the Polypedates leucomystax complex in

2 Thailand

3

4 Kittisak Buddhachat ${ }^{1, *}$ and Chatmongkon Suwannapoom ${ }^{2, *}$

5

$6 \quad{ }^{1}$ Department of Biology, Faculty of Science, Naresuan University, Phitsanulok, Thailand

$7 \quad{ }^{2}$ School of Agriculture and Natural Resources, University of Phayao, Phayao, Thailand

$8{ }^{*}$ These authors contributed equally to this work.

9

$10 *$ Corresponding authors

11 Kittisak Buddhachat

12 Email address: kittisakbu@,nu.ac.th, k_buddhachat@yahoo.com

13 Chatmongkon Suwannapoom

14 Email address: chatmongkonup@gmail.com

15

16

17

18

19

20

21 


\section{ABSTRACT}

24 Taxonomic uncertainty of the Asian tree frog Polypedates leucomystax complex presents the challenging task of inferring its biogeographical history. Here, we describe its dispersion and the genetic relationships among different populations in Thailand, where we connect the population of the P. leucomystax complex of the Sunda Islands to the Indochina (mainland) population based on analyses of 266 sequences of the mitochondrial cytochrome c oxidase subunit I (COI) gene. Our maternal genealogy implies that there are four well-supported lineages in Thailand, consisting of Northern A (clade A: Polypedates sp.), Nan (clade B: P. cf. impresus), Southern (clade C: P. cf. leucomystax) and Northern D (clade D: P. cf. megacephalus), with Bayesian posterior probability $>0.9$. Phylogeny and haplotype networks indicate that clades A, B and D are sympatric. In contrast, clade $\mathrm{C}(P$. cf. leucomystax $)$ and clade $\mathrm{D}(P$. cf. megacephalus $)$ are genetically divergent due to the geographical barrier of the Isthmus of Kra, resulting in an allopatric distribution. Climatic conditions, in particular differences in rainfall on each side of the Isthmus of Kra, may play an important role in limiting the immigration of both clades. For the within-populations of either clades $\mathrm{C}$ or $\mathrm{D}$, there was no significant correlation between geographic and genetic distance by the isolation-by-distance test, indicating intraspecificdispersal of each clade. Population expansion occurred in clade C, whereas clade D showed a constant population. Taken together, the P. leucomystax complex in South East Asia may have diversified under climatic pressure, leading to allopatric and/or sympatric speciation. 


\section{INTRODUCTION}

44 Southeast Asia contains a substantial genetic diversity of amphibians. Recent molecular

45 phylogenetic analyses have disclosed many anuran lineages that contain cryptic species.

46 Historically, complex changes in the region's geology and climate (e.g., Pleistocene climatic

47 oscillations) altered the topology and environmental conditions, resulting in an initial

48 fragmentation of habitat. These mechanisms generated the high species richness observed today

49 (Hall, 1998; Woodruff, 2010). Of interest to our research were the numerous frog species in

50 Southeast Asia whose taxonomy is still controversial, such as Microhyla fissipes (Yuan et al.,

51 2016), Staurois tuberilinguis (Matsui et al., 2007), Microhyla ornata (Matsui et al., 2005) and

52 Polypedates leucomystax (Kuraishi et al., 2013; Rujirawan, Stuart \& Aowphol, 2013). Accurate

53 species delimitation is essential to better understand their speciation and diversification and their

54 biogeography for conservation purposes.

The Asian tree frog, the P. leucomystax (Gravenhorst, 1829) complex, is an Asian

56 Rhacophoridae frog. These species are widely distributed in Southeast Asia, South China and

57 India. In addition, this species has phenotypic plasticity and exhibits high adaptation to the local

58 environment, leading to its existence in diverse habitats such as forests and even buildings. These

59 high levels of phenotypic plasticity present a great challenge for classification. Phylogenetic and

60 taxonomic relationships of the P. leucomystax complex throughout Southeast Asia exhibit

61 adaptive radiation (Kuraishi et al., 2013; Pan et al., 2013; Rujirawan, Stuart \& Aowphol, 2013).

62 At least six valid species, including $P$. braueri, $P$. impresus, $P$. leucomystax, $P$. macrotis, $P$.

63 megacephalus and $P$. mutus have been distinguished in the $P$. leucomystax complex based on

64 their morphology, advertisement calls and molecular data (Matsui, Seto \& Utsunomiya, 1986; 
65 Brown et al., 2010; Kuraishi et al., 2011; Kuraishi et al., 2013; Pan et al., 2013). Five species of 66 genus Polypedates, P. colletti, P. leucomystax, P. macrotis, P. megacephalus and P. mutus, can

67 be found in Thailand (Taylor, 1962; Heyer, 1971; Frost, 2013; Kuraishi et al., 2013; Pan et al., 68 2013; Rujirawan, Stuart \& Aowphol, 2013). Brown et al. (2010) indicated that much of the genetic divergence of the $P$. leucomystax complex was discovered in mainland rather than insular populations distributed throughout thousands of islands of the Malay Archipelago, presumably

71 resulting from range expansion mediated by transportation of agricultural products. Recently, a

72 new species, $P$. discantus, belonging to the $P$. leucomystax species complex from southern

73 Thailand was discovered using data on morphological characteristics, advertisement calls and

74 molecular evidence, which showed that this species was highly dissimilar to P. leucomystax and

75 P. megacephalus (Rujirawan, Stuart \& Aowphol, 2013). Several studies have confirmed the

76 existence of cryptic species of the P. leucomystax complex (Matsui, Seto \& Utsunomiya, 1986;

77 Kuraishi et al., 2011; Blair et al., 2013; Kuraishi et al., 2013; Pan et al., 2013).

78 In this study, we investigated the genetic variation, phylogenetic relationships and other

79 relevant factors that limit the dispersal of the P. leucomystax complex in Thailand. The present

80 results illustrate the range of distribution of putative $P$. leucomystax and putative $P$.

81 megacephalus, which is influenced by climatic conditions.

\section{MATERIALS AND METHODS}

\section{Sample collection, DNA extraction and sequencing}


85 A total of 266 adult $P$. leucomystax complex individuals were collected from 15 different

86 localities in Thailand (Table 1). All samples were dissected to obtain the liver, which was then

87 stored in absolute ethanol. Sample collection and euthanization were approved by the Center For

88 Animal Research Naresuan University under project number NU-AE591028. Genomic DNA

89 was extracted from liver tissue using a DNA extraction kit (RBC Bioscience, Singapore) and

90 kept at $-20{ }^{\circ} \mathrm{C}$ for further use. Individual DNA was used as a template for PCR amplification of

91 the mitochondrial COI gene using Taq DNA polymerase in a total volume of $25 \mu \mathrm{L}$ under the

92 following conditions: an initial denaturation at $94{ }^{\circ} \mathrm{C}$ for $5 \mathrm{~min}$, followed by $35-40$ cycles at 94

$93{ }^{\circ} \mathrm{C}$ for $30 \mathrm{~s}, 50{ }^{\circ} \mathrm{C}$ for $30 \mathrm{~s}$ and $72{ }^{\circ} \mathrm{C}$ for $1 \mathrm{~min}$, and a final extension step at $72{ }^{\circ} \mathrm{C}$ for $7 \mathrm{~min}$. PCR

94 products were visualized on $1.5 \%$ agarose gel under UV illuminator. The expected size of the

95 partial mitochondrial COI gene sequence was $688 \mathrm{bp}$. Subsequently, all PCR products were

96 purified using a QIAquick PCR Purification Kit (Qiagen, Germany) and then sequenced

97 (Macrogen, South Korea).

Phylogeny

100 Bayesian inference (BI) and maximum likelihood (ML) were employed for constructing a

101 phylogenetic tree based on the following partial COI sequences of the $P$. leucomystax complex

102 that were retrieved from GenBank: P. impresus: KP996822 (China), KP996846 (China),

103 KP087862-70 (Laos); P. leucomystax: KR087871-2 (Thailand); and P. megacephalus:

104 KR087879, KR087881 (Thailand). First, the best-fit model of DNA sequence evolution for this

105 locus was identified with the Akaike information criterion (AIC) implemented in MrModeltest

106 v2.3 (Nylander, 2004), resulting in the GTR $+\mathrm{I}+\mathrm{G}$ model as the best fit with AIC Subsequently, a 
107 Bayesian tree was constructed based on the base substitution calculated from the $\mathrm{GTR}+\mathrm{I}+\mathrm{G}$

108 model through MrBayes 3.1.2 (Ronquist \& Huelsenbeck, 2003) with two independent searches

109 with random starting trees for 5 million generations, in which the diagnostic was calculated

110 every 1,000 generations and compared using four Markov chain Monte Carlo chains (temp $=$

111 0.2). The log-likelihood scores were used for plotting the convergence in Tracer v1.5 (Rambaut

112 et al., 2013) and building a consensus tree, which was completed by the removal of the first $25 \%$

113 of the generations from each run. For maximum likelihood analysis, RAxML 7.0.4 was carried

114 out (Stamatakis, Hoover \& Rougemont, 2008) using the GTR+I+G model for nucleotide

115 substitution (same as the BI analysis) with 1,000 bootstrap replicates.

\section{Population genetics and structure}

118 A total of 266 sequences of mitochondrial COI were aligned using ClustalW (implemented in

119 MEGA 6.0 with default parameters). The number of polymorphic sites, the parsimony-

120 informative sites, singleton sites, the number of haplotypes, haplotype diversity $\left(H_{\mathrm{d}}\right)$, and

121 nucleotide diversity for each clade were calculated using DnaSP v5.0 (Librado \& Rozas, 2009).

122 Genetic distances among taxa were calculated using the corrected $p$-distance model in MEGA

1236.0 (Tamura et al., 2011). Furthermore, we detected a boundary line in the genetic landscape

124 between the Northern D clade and the Southern clade using Barrier 2.2 (Manni, Guérard \&

125 Heyer, 2004). A minimum spanning network was constructed using PopART (Population

126 Analysis with Reticulate Trees) to define the relationships among haplotypes and the distribution

127 of haplotypes in each locality (Bandelt, Forster \& Röhl, 1999). To evaluate the effect of 
128 geographic distance on the genetic divergence among populations of the Northern D clade and

129 among populations of the Southern clade, a linear regression model was carried out.

\section{Demographic history}

132 To investigate the demographic history of $P$. megacephalus and $P$. leucomystax populations in

133 Thailand, multiple approaches were explored using DnaSP (Librado \& Rozas, 2009). Neutrality

134 tests of Tajima's $D$ (Tajima, 1989) and Fu's Fs (Fu, 1997) for the two species were completed. A

135 significantly positive value indicates a process of subdivision or a recent population bottleneck,

136 whereas a population expansion results in a significantly negative value. Pairwise mismatch

137 distribution was used assuming a constant population size (Roger \& Harpendin, 1992).

138 Multimodal mismatch distribution implies stability of the population, while unimodal mismatch

139 distribution reflects an expanding population. In addition to these methods, the raggedness index

$140\left(r_{\mathrm{g}}\right)$ of the observed distribution was calculated (Harpending, 1994). A small $r_{g}$ indicates a

141 demographic expansion.

142

\section{RESULTS}

\section{Sequence characteristics}

145 A total of 266 samples of the $P$. leucomystax complex yielded $688 \mathrm{bp}$ fragments of the

146 mitochondrial COI gene. All new sequences in this study were deposited in the GenBank

147 database (MG583020-MG583285). After multiple alignment of all COI sequences, the sequences 
148 were trimmed to the same length $(437 \mathrm{bp})$ before downstream analysis. We observed 82

149 polymorphic sites, which are also 82 parsimony-informative sites without a singleton site,

150 resulting in the acquisition of 15 haplotypes (Table 2). Overall nucleotide and haplotype

151 diversity were 0.0664 and 0.9000 , respectively (Table 2 ).

152

153 Phylogenetic analyses and haplotype distribution

154 Based on 266 mitochondrial COI sequences of the P. leucomystax complex, a matrilineal

155 genealogy was generated, and our results indicated that the P. leucomystax complex in Thailand

156 consists of four clades: clade A (Northern A), Polypedates sp.; clade B (Nan), P. cf. impresus;

157 clade C (Southern), P. cf. leucomystax; and clade D (Northern D), P. cf. megacephalus (Fig. 1).

158 With respect to phylogenetic inference, clade A was treated as a sister group of clade B, which

159 was found in Nan. Clade A, however, can be seen in genetic samples obtained from the

160 Kanchanaburi (KCB), Mae Hong Son (MHS) and Phetchaburi (PCB) provinces and shared a

161 habitat with clade D, which was recognized as $P$. cf. megacephalus; its distribution range was in

162 the far north of the Isthmus of Kra at Chiang Mai (CM), MHS, KCB, PCB, Saraburi (SRB), Loei

163 (LPR), Nakhon Ratchasima (NRS) and Prachuap Khiri Khan (PKK). The dispersal areas of clade

$164 \mathrm{C}$, as represented by $P$. cf. leucomystax, included Chumphon (CP), Nakhon Si Thammarat

165 (NST), Phuket (PK) and Ranong (RN), which are south of the Isthmus of Kra (Fig. 2A). Barrier

1662.2 was employed to determine a barrier for immigration between clade C and clade D

167 populations based on the dataset of genetic distance (Kimura's two-parameter model). Likely,

168 the Isthmus of Kra (IOK) represents a significant barrier to restrict immigration based on the

169 great genetic distance that was noted around IOK (Fig. 2B) 
171 as shown in Fig. 1. Clade D exhibited the highest number of haplotypes at seven $\left(H_{\mathrm{d}}=0.746\right)$,

172 followed by clade $\mathrm{C}$ with five haplotypes $\left(H_{\mathrm{d}}=0.7526\right)$ (Table 2$)$. Haplotypes $\mathrm{A}$ and $\mathrm{B}$, seen in

173 clade A, and haplotype C found in clade B were unique haplotypes. Haplotypes D-I were noted

174 in clade $\mathrm{D}$, while populations of northern, western and upper southern Thailand (KCB, $\mathrm{PCB}$ and

175 PKK, respectively) shared haplotype J. Haplotypes F and G of clade D are considerably

176 divergent from the rest of clade $\mathrm{D}$ and we partition it in two subclades: D1 and D2. Clade $\mathrm{C}(P$.

177 cf. leucomystax) had high haplotype diversity and contained three unique haplotypes (M, N and

178 O) and two shared haplotypes (K and L).

179 Analyses of the linear regression model between the genetic distance of the mitochondrial

180 COI gene sequence and the geographical distance found no significant isolation-by-distance

181 effect among populations of clade C (P. cf. leucomystax) and clade D (P. cf. megacephalus) (Fig. $1823)$.

\section{Demographic history}

184 When we defined a significant barrier around the Isthmus of Kra (IOK) leading to the genetic

185 divergence between clade C (P. cf. leucomystax) and clade D (P. cf. magacephalus), neutrality

186 tests (Tajima's $D$ and Fu's $F s$ ) of both species were not significantly positive, whereas Fu's $F s$ of

187 clade C was significantly positive (Table 3). Furthermore, the mismatch distribution was tested

188 as a result of a left-skewed multimodal mismatch distribution for clade $\mathrm{D}$ with moderate $r_{\mathrm{g}}$

189 (0.2031) but a unimodal mismatch distribution for clade $\mathrm{C}$ with a low $r_{\mathrm{g}}(0.0569)$ (Fig. 4).

190 Overall, these results suggested a constant population size of clade D and a population expansion 191 of clade C. 


\section{DISCUSSION}

194 The taxonomy of the Asian tree frog of the P. leucomystax complex is contentious due to the 195 species' widespread distribution from Nepal to South East Asia and similar morphologies. To

196 better understand the population structure and biogeography of the P. leucomystax complex in

197 Thailand, where there is a substantial area for their genetic dispersal, the COI mitochondrial gene

198 sequences of these species were analysed. Our matrilineal genealogy implied four well-supported

199 lineages, consisting of a Northern D clade (clade D), a Southern clade (clade C), a Nan clade

200 (clade B) and a Northern A clade (clade A); based on their sequences and distributions, they

201 might be treated as $P$. megacephalus, $P$. leucomystax, $P$. impresus and Polypedates sp.,

202 respectively.

Although the mtDNA phylogeny reveals the presence of distinct clades, mtDNA

204 introgression is frequently observed where closely related species are in secondary contact

205 (Toews and Brelsford, 2012, Zieliński et al., 2013, Wielstra et al., 2017a and Wielstra et al., 206 2017b). This can occur particularly in sympatric populations or at the boundaries of species

207 distributions resulting from incomplete reproductive isolation. For instance, a pair of crested

208 newt species (genus Triturus) form a hybrid zone (south of the Marmara Sea), where

209 introgression suggests movement of the hybrid zone (Wielstra et al., 2017b). Therefore, the use

210 of mitochondria DNA data alone is not sufficient for species delineation. An integrative

211 approach, consulting additional data such as morphology, bioacoustics, ecology, and/or nuclear

212 DNA, would be required to more accurately assess the taxonomy of the P. leucomystax complex

213 (Padial et al., 2010). Kuraishi et al., (2013) found one sample of the P. leucomystax complex 
214 from Indochina shared nuclear brain-derived neurotrophic factor (BDNF) and recombination

215 activating gene 1 (RAG-1) haplotypes with one sample from the south of IOK, possibly

216 suggesting deep coalescence or incomplete lineage sorting.

217 Bayesian inference strongly supported the geographic distinction of species in the

218 Northern D clade and the Southern clade. Furthermore, Monmonier's algorithm suggested that

219 the Isthmus of $\mathrm{Kra}$ (IOK; located between 11 and $13{ }^{\circ} \mathrm{N}$ along the Thai Peninsula) corresponds

220 to a considerable phylogeographic break as evidenced by the large genetic divergence between

221 the populations on either side of the isthmus. Kuraishi et al. (2013) explored the population

222 structure of $P$. leucomystax complex in Southeast Asia and revealed that $P$. megacephalus is

223 restricted to Indochina (the northern Thailand, Laos, Vietnam and southern China) (Kuraishi et

224 al., 2013; Pan et al., 2013), while the range of P. leucomystax is restricted to the Sunda regions

225 (southern Thailand and Malay Archipelago) (Brown et al., 2010; Kuraishi et al., 2013). Based on

226 their distribution, we ascribe the Northern D clade to P. megacephalus and the Southern clade to

227 P. leucomystax. The Isthmus of Kra has been well-characterized as a considerable biogeographic

228 boundary of both faunal and floral assemblages that are limited on either side. Bruyn et al (2005)

229 used genetic evidence from Macrobrachium rosenbergii (freshwater shrimp with a broad

230 distribution) to hypothesize that the existence of a seaway across the IOK over a million year

231 contributed to its geographically discontinuous distribution. In contrast, Hughes et al. (2011),

232 based on marine fossils and geological data, proposed that the occurrence of species with distinct

233 distributions in the north and south of the IOK is related to the influence of the climatic zone

234 rather than either marine inundation or breaches at any point near the IOK. In addition, flying

235 animals such as birds and bats also have a restricted species distribution which is bounded by the

236 IOK (Hughes et al.,2011). This indicates that the geophysical barriers at the IOK, such as the 
237 waterway, are unlikely to be significant biogeographical boundaries causing vicariance of 238 various species at the IOK. Hughes et al. (2011) also determined the climatic zone in Indochina 239 and the Malay Peninsula is the result of four climatic zonations, which are displayed as similar 240 climatic zones found in each time period from the Last Interglacial Period to the current 241 conditions. Of interest is the climatic divergence in precipitation, temperature and seasonality

242 between the central zone (north of IOK) and the southern zone (south of IOK). Based on this, we 243 suggest climate was a significant factor in shaping the spatial distribution of the genetic lineage

244 between the Northern and Southern clades. The haplotype network also shows that the Northern 245 D clade and the Southern clade have the highest genetic diversity and a wide range distribution 246 in Thailand, although the most recent divergence time between P. megacephalus (putative 247 Northern D clade) and P. leucomystax (putative Southern clade) was estimated to be in the late 248 Pliocene or early Pleistocene (1.4-4.0 MYBP) (Kuraishi et al., 2013). We assumed that the 249 populations of the Southern clade (putative P. leucomystax) expanded and colonized the northern 250 part of Indochina, leading to the diversification of the species. This hypothesis is partially 251 supported by the results of the unimodal pairwise difference and the small value of the 252 raggedness index in the Southern clade (Fig. 3). Within the population of the Northern D clade, maternal genealogy demonstrated that the 254 genetic samples from Nakhon Ratchasima province (NRS) seemed to represent a naturally 255 occurring divergence because of the emergence of endemic haplotypes; however, it was a lowsupported lineage, with 0.7 Bayesian posterior probability (BPP). When we considered the topography of this region, the population of NRS as clade D1 is partitioned from the other populations within clade D by the two mountain ranges, Dong Phaya Yen and Sankamphaeng.

259 This may be a possible barrier to gene flow among the modern populations of Northern D 
260 between eastern (clade D1) and western (clade D2; NRS) Thailand. Unfortunately, only a limited

261 number of populations from the east of Thailand were investigated in this study. Further work for

262 validating whether Dong Phaya Yen and Sankamphaeng are a barrier to gene flow in $P$.

263 megacephalus is required. According to the demographic history, the population of Northern D

264 was a stable population, but the population of Southern clade showed a relatively similar

265 unimodal distribution with a small raggedness index, possibly indicating a population expansion.

266 This result was similar to that observed in the population of $P$. leucomystax in the northern

267 Philippines (Brown et al., 2010). This scenario implied a genetically homogenous population,

268 especially in the population of Phuket Island, which shared a haplotype with NST, probably

269 caused by a recent population expansion due to the founder effect. Although the population of

270 the Northern D clade expanded, it was limited to localities south of the Isthmus of Kra.

\section{CONCLUSIONS}

272 Our matrilineal genealogy of the P. leucomystax complex in Thailand suggested four lineages,

273 i.e., Nan (putative $P$. impresus), Northern D (putative $P$. megacephalus), Southern (putative $P$.

274 leucomystax) and Northern A (Polypedates sp.) clades. We noted that the populations of the

275 Northern D, Nan and Polypedates sp. clades are in sympatry, while their distributions are

276 allopatric to the southern clade (P. leucomystax) due to the separation by the Isthmus of Kra.

277 Climatic conditions may be a major contributor to limited migration of the current populations of

278 both clades, but climatic oscillation in the Pliocene and Pleistocene is a highly possible scenario

279 that drove speciation resulting in diversification of the $P$. leucomystax complex in Southeast Asia

280 and China, which includes the divergence of the southern and northern clades in Thailand. 
282 Bandelt HJ, Forster P, Röhl A. 1999. Median-joining networks for inferring intraspecific 283 phylogenies. Molecular Biology and Evolution 16:37-48.

284

285

286

287

288

289

290

291

292

293

294

295

296

297

298

299

300

301

302

303

304

Blair C, Davy CM, Ngo A, Orlov NL, Shi HT, Lu SQ, Gao L, Rao DQ, Murphy RW. 2013. Genealogy and demographic history of a widespread amphibian throughout Indochina. Journal of Heredity 104:72-85.

Brown RM, Linkem CW, Siler CD, Sukumaran J, Esselstyn JA, Diesmos AC, Iskandar DT, Bickford D, Evans BJ, McGuire JA, Grismer L, Supriatna J, Andayani N. 2010. Phylogeography and historical demography of Polypedates leucomystax in the islands of Indonesia and the Philippines: evidence for recent human-mediated range expansion? Molecular Phylogenetics and Evolution 57:598-619.

de Bruyn M, Nugroho E, Hossain MM, Wilson JC, Mather PB. 2005. Phylogeographic evidence for the existence of an ancient biogeographic barrier: the Isthmus of Kra Seaway. Heredity 94: 370-378.

Frost DR. 2013. Amphibian species of the world: an online reference. Version 6.0. New York: American Museum of Natural History. [electronic database] Available at http://research.amnh.org/herpetology/amphibia/index.html (accessed 22 June 2017).

Fu YX. 1997. Statistical tests of neutrality of mutations against population growth, hitchhiking and background selection. Genetics 147: 915-925.

Hall R. 1998. The plate tectonics of Cenozoic SE Asia and the distribution of land and sea. In: Hall R, Holloway JD, eds. Biogeography and geological evolution of SE Asia. Leiden, The Netherlands: Backhuys Publishers, 99-131.

Harpending HC. 1994. Signature of ancient population growth in a low-resolution mitochondrial DNA mismatch distribution. Human Biology 591-600. 
305 Heyer WR. 1971. Mating calls of some frogs from Thailand. Chicago: Field Museum of Natural 306 History.

307 Hughes AC, Satasook C, Bates PJ, Bumrungsri S, Jones G. 2011. Explaining the causes of 308 the zoogeographic transition around the Isthmus of Kra: using bats as a case study.

$309 \quad$ Journal of Biogeography 38: 2362-2372.

310 Kuraishi N, Matsui M, Hamidy A, Belabut DM, Ahmad N, Panha S, Sudin A, Yong HS,

311 Jiang JP, Ota H, Thong HT, Nishikawa K. 2013. Phylogenetic and taxonomic

312 relationships of the Polypedates leucomystax complex (Amphibia). Zoologica Scripta

$313 \quad$ 42:54-70.

314 Kuraishi N, Matsui M, Ota H, Chen S-L. 2011. Specific separation of Polypedates braueri

315 (Vogt, 1911) from P. megacephalus (Hallowell, 1861) (Amphibia: Anura:

316 Rhacophoridae). Zootaxa 2744:53-61.

317 Librado P, Rozas J. 2009. DnaSP v5: a software for comprehensive analysis of DNA

318 polymorphism data. Bioinformatics 25:1451-1452.

319 Manni F, Guérard E, Heyer E. 2004. Geographic patterns of (genetic, morphologic, linguistic)

320 variation: how barriers can be detected by using Monmonier's algorithm. Human Biology

$321 \quad 76: 173-190$.

322

323

324

325

326

327

\section{Matsui M, Ito H, Shimada T, Ota H, Saidapur SK, Khonsue W, Tanaka-Ueno T, Wu GF.} 2005. Taxonomic relationships within the pan-oriental narrow-mouth toad Microhyla ornata as revealed by mtDNA analysis (Amphibia, Anura, Microhylidae). Zoological Science 22:489-495.

Matsui M, Mohamed M, Shimada T, Sudin A. 2007. Resurrection of Staurois parvus from $S$. tuberilinguis from Borneo (Amphibia, Ranidae). Zoological Science 24:101-106. 
328 Matsui M, Seto T, Utsunomiya T. 1986. Acoustic and karyotypic evidence for specific separation of Polypedates megacephalus from P. leucomystax. Journal of Herpetology

330 20:483-489.

331 Nylander JA. 2004. MrModeltest v2.3. [program distributed by the author] Uppsala, Sweden: Evolutionary Biology Centre, Uppsala University.

Padial JM, Miralles A, De la Riva I, Vences M. 2010. The integrative future of taxonomy. Frontiers in Zoology 7:16.

Pan S, Dang N, Wang J, Zheng Y, Rao D, Li J. 2013. Molecular phylogeny supports the validity of Polypedates impresus Yang 2008. Asian Herpetological Research 4:124-133.

Rambaut A, Suchard MA, Xie D, Drummond AJ. 2013. Tracer v1.5. Available at http://beast.bio.ed.ac.uk/Tracer (accessed 22 June 2017).

Rogers AR, Harpending H. 1992. Population growth makes waves in the distribution of pairwise genetic differences. Molecular Biology and Evolution 9: 552-569.

Ronquist F, Huelsenbeck JP. 2003. MrBayes 3: Bayesian phylogenetic inference under mixed models. Bioinformatics 19:1572-1574.

Rujirawan A, Stuart BL, Aowphol A. 2013. A new tree frog in the genus Polypedates (Anura: Rhacophoridae) from southern Thailand. Zootaxa 3702:545-565.

Stamatakis A, Hoover P, Rougemont J. 2008. A rapid bootstrap algorithm for the RAxML web servers. Systematic Biology 57:758-771.

Tajima F. 1989. Statistical method for testing the neutral mutation hypothesis by DNA polymorphism. Genetics 123: 585-595. 
349 Tamura K, Stecher G, Peterson D, Filipski A, Kumar S. 2013. MEGA6: Molecular

350 Evolutionary Genetics Analysis version 6.0. Molecular Biology and Evolution 30:2725-

3512729.

352 Taylor EH. 1962. The amphibian fauna of Thailand. University of Kansas Science Bulletin 43:265-599.

Toews DP, Brelsford A. 2012. The biogeography of mitochondrial and nuclear discordance in 355 animals. Molecular Ecology 21:3907-3930.

Wielstra B, Burke T, Butlin RK, Arntzen JW. 2017a. A signature of dynamic biogeography: enclaves indicate past species replacement. Proceedings of the Royal Society B 284:20172014

Wielstra B, Burke T, Butlin RK, Avcı A, Üzüm N, Bozkurt E, Olgun K, Arntzen JW. 2017b. A genomic footprint of hybrid zone movement in crested newts. Evolution Letters 1:93-101. DOI:10.1002/ev13.9.

Woodruff DS. 2010. Biogeography and conservation in Southeast Asia: how 2.7 million years of repeated environmental fluctuations affect today's patterns and the future of the remaining refugial-phase biodiversity. Biodiversity and Conservation 19:919-941. Murphy RW, Che J. 2016. Red River barrier and Pleistocene climatic fluctuations shaped the genetic structure of Microhyla fissipes complex (Anura: Microhylidae) in southern China and Indochina. Current Zoology 62:531-543. 
371 complete mtDNA replacement in the Carpathian newt (Lissotriton montandoni). Molecular Ecology 22:1884-1903. 


\section{Figure 1}

Phylogeographic relationships and a minimum spinning network of all haplotype of the Polypedates leucomystax complex among populations in Thailand.

It consists of clade A as Polypedates sp., clade B as P. cf. impresus, clade C (the Southern clade) as $P$. cf. leucomystax, and clade $D$ (the Northern D clade) as $P$. cf. megacephalus, as well as outgroups (KR087858, KP996762 $=$ P. braueri) inferred from Bayesian analysis of mitochondrial COI gene sequences. Bayesian posterior probability values are expressed above internodes. The asterisks above branches represent bootstrap support for Bayesian posterior probabilities and maximum likelihood (>95\%). Scale bar represents 0.5 nucleotide substitutions per site. IOK represents the Isthmus of Kra.

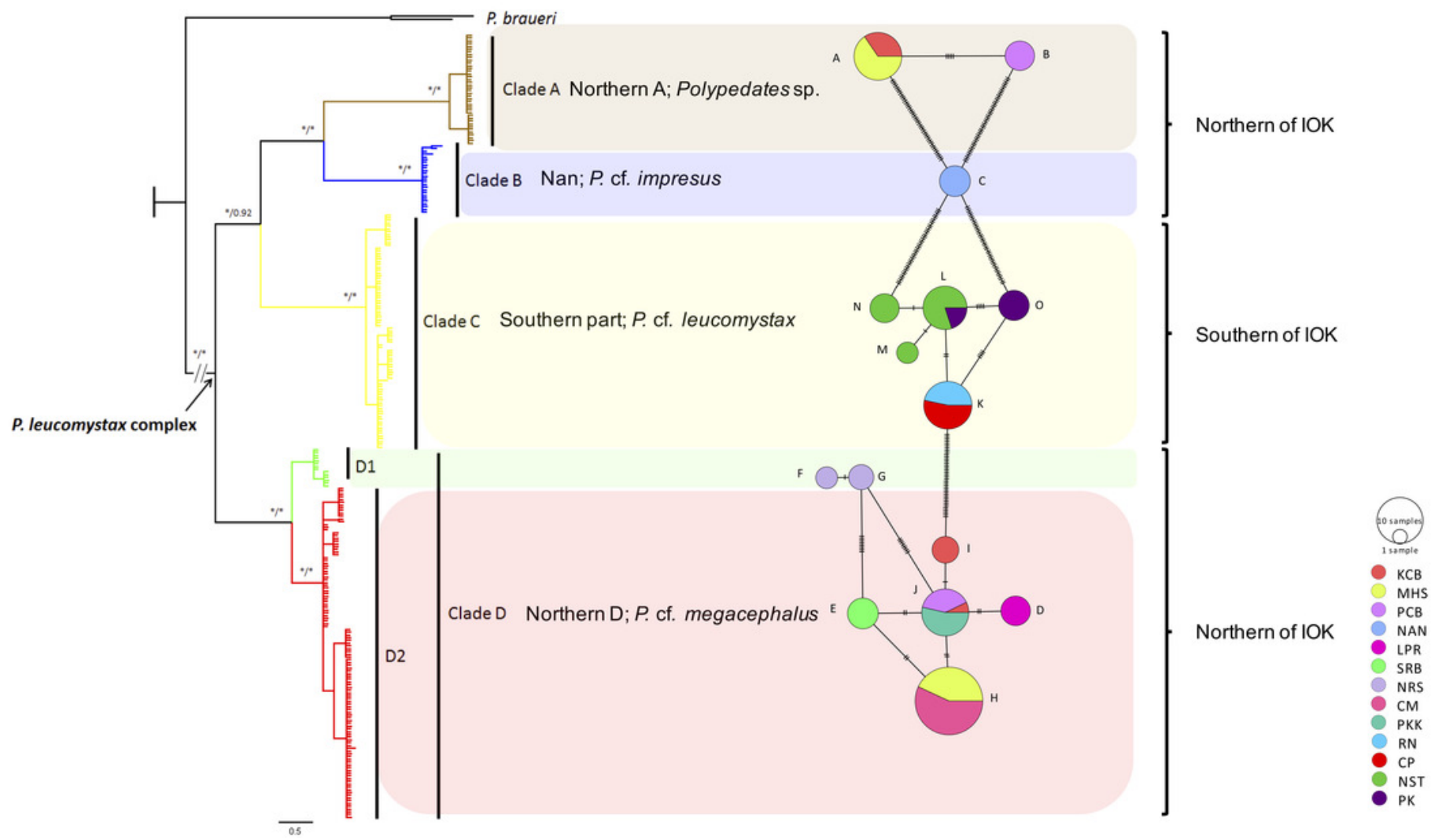


Figure 2

Clade distribution of the $P$. leucomystax complex throughout Thailand $(A)$ and the genetic differentiation across the Northern $D$ clade (putative $P$. megacephalus; PM) and the Southern clade (putative $P$. leucomystax; PL) (B).

The abbreviations for each locality are given in Table 1. Different colors represent the different clades. "a" represents a significant barrier to partition the distribution of the two clades, by Barrier version 2.2.

A

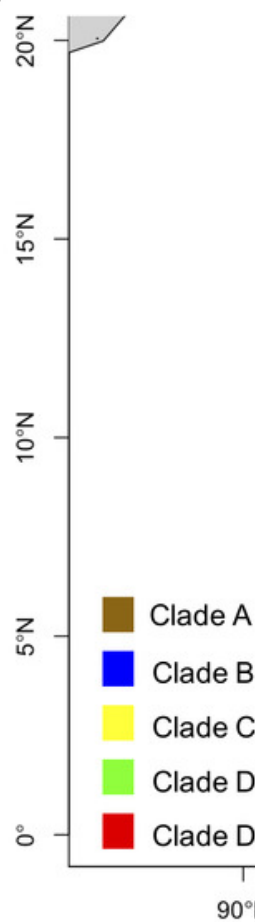

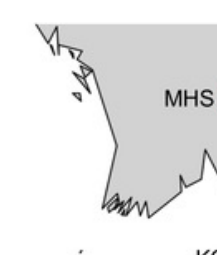

$$
8
$$<smiles>c1ccc(C23CC4CC(CC(C4)C2)C3)cc1</smiles>$$
\text { . }
$$$$
\text { . }
$$$$
\text { ' }
$$$$
\mathrm{RN}
$$

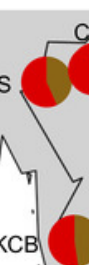

che

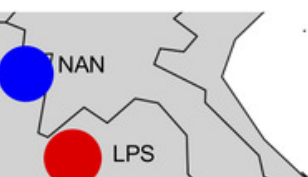

LPS

of
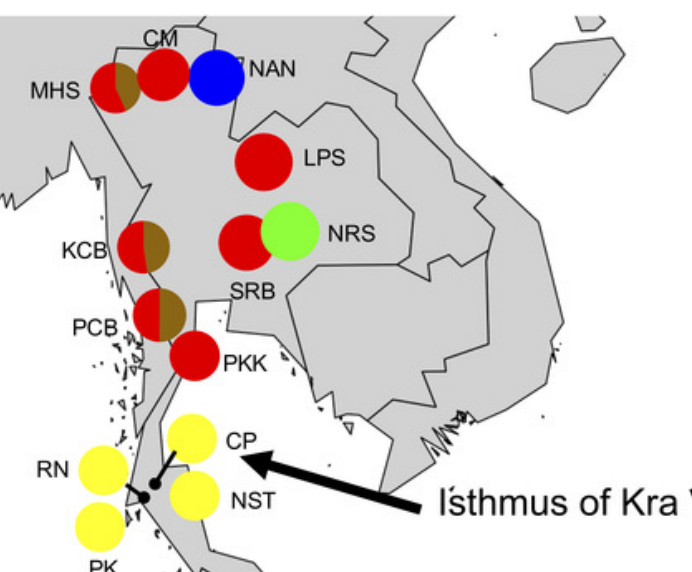

B

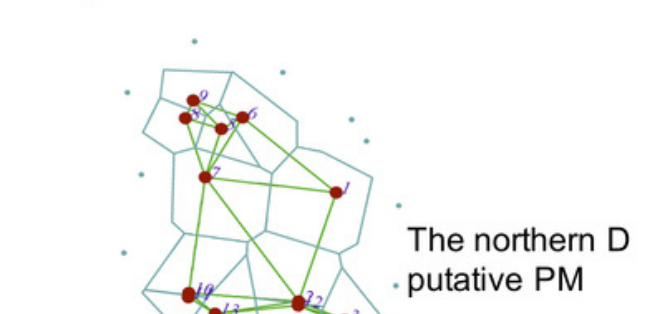


Figure 3

The correlation of genetic distance and linear geographic distance $(\mathrm{km})$ for $(\mathrm{A})$ the Northern D clade (putative Polypedates megacephalus) and (B) the Southern clade (putative Polypedates leucomystax).
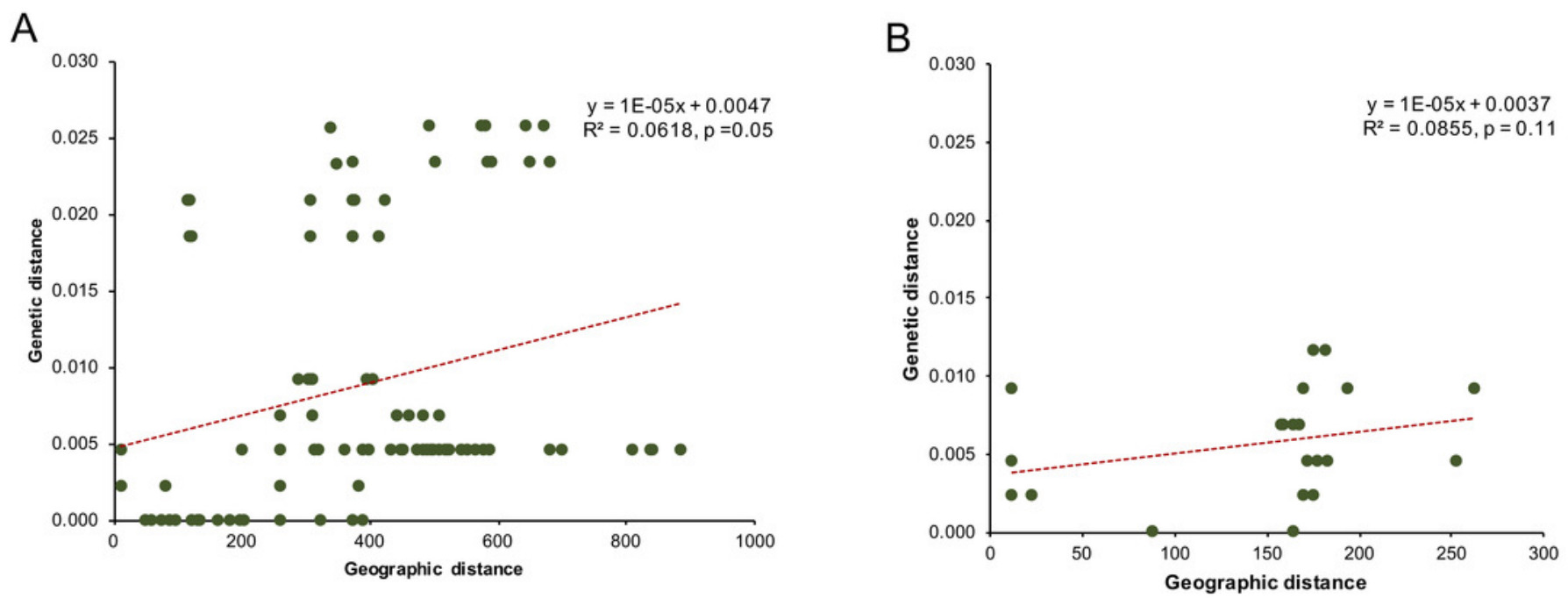


\section{Figure 4}

Mismatch distribution of the mitochondrial COI gene in $(A)$ the Northern $D$ clade (putative Polypedates megacephalus) and (B) the Southern clade (putative Polypedates leucomystax).

The raggedness $(\mathrm{rg})$ index is calculated to evaluate the population expansion of each species. Ramos-Onsins and Rozas's R2 statistic represents the population growth.

A

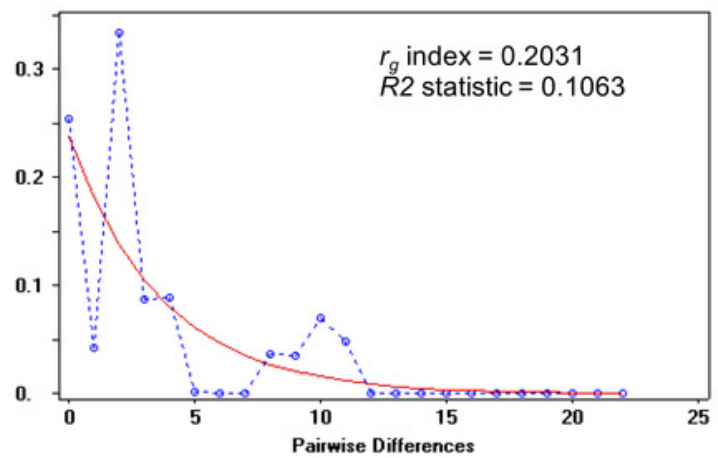

B

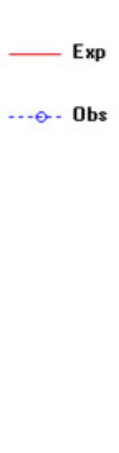

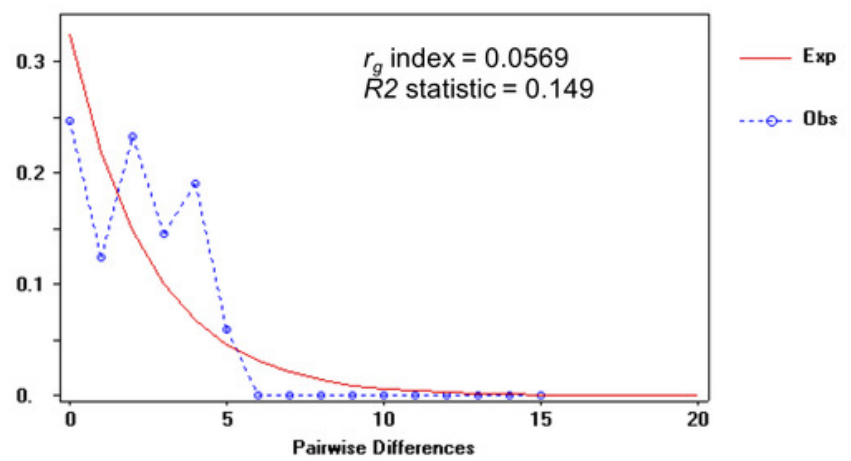




\section{Table $\mathbf{1}$ (on next page)}

Localities of sample collection for the Polypedates leucomystax complex in Thailand. 
1 Table 1 Localities of sample collection for Polypedates leucomystax complex in Thailand.

\begin{tabular}{lccccc}
\hline \multicolumn{1}{c}{ Locality } & Abbreviation & Number & Altitude (m above sea level) & Longitude & Latitude \\
\hline Nan province & NAN & 12 & 665 & 18.980974 & 101.182594 \\
Kanchanaburi province & KCB & 20 & 917 & 14.69329 & 98.40535 \\
Loei province: Phu Ruea & LPR & 11 & 939 & 17.48193 & 101.34982 \\
Nakhon Ratchasima province & NRS & 14 & 865 & 14.49336 & 101.87364 \\
Chiang Mai province: Mae Wang & CM & 7 & 678 & 18.657305 & 98.681831 \\
Chiang Mai province: Doi Saket & CM & 13 & 402 & 18.98777 & 99.11455 \\
Chiang Mai province: Omkoi & CM & 13 & 460 & 17.47137 & 98.45785 \\
Mae Hong Son province & MHS & 44 & 396 & 19.24797 & 97.99542 \\
Saraburi province & SRB & 12 & 105 & 14.70993 & 100.81819 \\
Phetchaburi province & PCB & 22 & 329 & 14.70993 & 100.81819 \\
Prachuap Khiri Khan province & PKK & 15 & 23 & 11.43678 & 99.56011 \\
Ranong province & RN & 14 & 18 & 9.6052 & 98.4669 \\
Nakhon Si Thammarat province & NST & 37 & 97 & 8.76902 & 99.80349 \\
Phuket province: Thalang & PK & 17 & 31 & 7.96804 & 98.33589 \\
Chumphon province & CP & 15 & 103 & 10.110278 & 99.082778 \\
\hline
\end{tabular}




\section{Table 2 (on next page)}

Summary of the $P$. leucomystax complex in Thailand

Major lineages clades, putative scientific name, number of individuals $(N)$, number of mtDNA haplotypes $(n)$, number of polymorphic sites $(\mathrm{P})$, parsimony-informative sites $(\mathrm{PI})$ and singleton sites $(\mathrm{S})$, haplotype diversity $\left(H_{\mathrm{d}}\right)$ and nucleotide diversity $(\pi)$. 
Table 2 Summary of the P. leucomystax complex in Thailand: major lineages clades, putative scientific name, number of individuals $(N)$, number of mtDNA haplotypes $(n)$, number of polymorphic sites $(\mathrm{P})$, parsimony-informative sites $(\mathrm{PI})$ and singleton sites $(\mathrm{S})$, haplotype diversity $\left(H_{\mathrm{d}}\right)$ and nucleotide diversity $(\pi)$.

\begin{tabular}{lllllllll}
\hline Clade & Putative species & $N$ & $n$ & \multicolumn{1}{c}{$\pi$} & $H_{\mathrm{d}}$ & $\mathrm{P}$ & $\mathrm{S}$ & PI \\
\hline A (the Northern A) & Polypedates sp. & 40 & 2 & 0.0037 & 0.4089 & 4 & 0 & 4 \\
B (Nan) & P. impresus & 12 & 1 & 0 & 0 & 0 & 0 & 0 \\
C (the Southern) & P. megacephalus & 131 & 7 & 0.0048 & 0.746 & 15 & 1 & 14 \\
D (the Northern D) & P. leucomystax & 83 & 5 & 0.0073 & 0.7526 & 7 & 0 & 7 \\
Total & & 266 & 15 & 0.0664 & 0.9 & 82 & 0 & 82 \\
\hline
\end{tabular}




\section{Table 3(on next page)}

Summary of statistics used to compute the demographic history of populations of the Northern D clade (putative $P$. megacephalus) and the Southern clade (putative $P$. leucomystax). 
Table 3 Summary of statistics used to compute the demographic history of populations of the Northern B clade (putative P. megacephalus) and the Southern clade (putative $P$. leucomystax).

\begin{tabular}{lclcc}
\hline & \multicolumn{2}{c}{ Tajima's $D$} & \multicolumn{2}{c}{ Fu's $F s$} \\
\cline { 2 - 5 } Clade & $D$ & $P$ value & $F s$ & $P$ value \\
\hline The Northern D & 0.439 & $>0.1$ & 3.213 & 0.045 \\
The Southern & 1.176 & $>0.1$ & 3.031 & 0.071 \\
\hline
\end{tabular}

